\title{
Altered Electromyographic Activity Pattern of Rat Soleus Muscle Transposed into the Bed of Antagonist Muscle
}

\author{
Urszula Slawinska and Stefan Kasicki \\ Neurophysiology Department, Nencki Institute of Experimental Biology, 02-093 Warsaw, Poland
}

Human patients suffering from motor paralysis of the leg can learn, to some extent, how to use the transposed antagonistic muscle in place of the damaged or ineffective muscle. Experiments on animals showed opposite results, although in a few experiments the functional reorganization of the activity of the transposed muscle was not excluded. In our experiments, we performed transposition of the soleus (Sol) with a preserved innervation into the bed of the removed extensor digitorum longus (EDL) in 6-d-old pups and 3-month-old rats. The locomotor and reflex EMG activity of the transposed Sol (trSol) was recorded in chronic experiments 3-4 months later. Our results showed that the EMG activity of the Sol might be modified when the muscle is transposed into the bed of the antagonistic muscle EDL. The modification consisted of an additional burst of activity during the swing phase and of reflex response of the muscle to the plantar flexion. This modification was present in all animals operated on at $6 \mathrm{~d}$ of age and in two of six adults. After chronic experiments, we excluded the possibility that the flexor-like activity was induced by additional innervation of the trSol by the cut end of the EDL nerve. We suggest that the observed modifications of activity of trSol might be caused by readjustment of the spinal network organization to the new functional demands. Two factors might be responsible for this readjustment: the immaturity of the nervous system at the moment of surgery and preserved afferent innervation of the transposed muscle.

Key words: muscle transposition; change of EMG activity; development; chronic EMG; locomotor EMG pattern; rat
The experimental animal models of functional recovery attributable to the plasticity of neuronal connections are primarily based on various sensory systems. Animal experiments investigating functional plasticity within the motor system did not show clear evidence of readjustment of motor function after tendon transfers or muscle transpositions.

It was shown in the cat forelimb that transfer of the muscle tendon to its antagonist muscle partially reversed the movements at the joint (Missiuro and Kozlowski, 1963; Yumiya et al., 1979). The animals gradually developed the proper pattern during locomotor behavior. Thus, there was an active relearning of motor coordination after cross-connection of forearm muscles, as seen from clinical observations. In the hindlimb of the cat, the effect of muscle transposition was studied on the gastrocnemius muscle (Forssberg and Svatengren, 1983). The authors concluded that neither the spinal locomotor network controlling the gastrocnemius muscle nor the supraspinal circuits influencing the network exhibited a high degree of plasticity when the muscle was in a position antagonistic to its original state.

The experiments mentioned above were performed on adult animals, but the best substrate for plastic changes responsible for functional reorganization of the neural network is the immature nervous system. Thus, to clarify the impact of the age at which the transposition of the muscle was performed, we performed experiments on pups and adult hooded rats. The project was approved

Received Feb. 20, 2002; revised April 29, 2002; accepted May 3, 2002.

This paper was supported by State Committee for Scientific Research Grant 4P05A09317 and by statutory funds from the Nencki Institute of Experimental Biology.

Correspondence should be addressed to Urszula Slawinska, Neurophysiology Department, Nencki Institute of Experimental Biology, 3 Pasteur Street, 02-093 Warsaw, Poland. E-mail: ulaslaw@nencki.gov.pl.

Copyright (C) 2002 Society for Neuroscience $\quad 0270-6474 / 02 / 220001-05 \$ 15.00 / 0$ by the Local Ethics Committee at the Nencki Institute and followed European Union guidelines on animal care.

\section{MATERIALS AND METHODS}

\section{Chronic experiments}

Muscle transposition. The experiments were performed on two groups of animals: in group A, transposition of the soleus (Sol) muscle was performed on seven immature pups ( $6 \mathrm{~d}$ of age), which were returned after surgery to their mothers; in group B, transposition of the Sol was performed on six adult animals ( 3 months of age). The fully aseptic surgery was performed either under ether anesthesia (group A) or under Equithesin anesthesia (group B; $0.4 \mathrm{ml} / 100$ gm body weight). The skin on the left hindlimb was opened, and the Sol and extensor digitorum longus (EDL) muscles were gently exposed (Fig. $1 A$ ). The proximal and distal tendons of the Sol were dissected and cut, and the entire muscle was moved over into the bed of the EDL. The nerve and associated vascular bundle were left intact. The proximal and distal tendons of the transposed Sol (trSol) were sutured (Ethicon 8/0; Ethicon Ltd., Edinburgh, $\mathrm{UK}$ ) to the corresponding tendons of the EDL (Fig. 1B). After sewing on both tendons of the trSol, the main part of the EDL ( $\sim 82-88 \%$ of muscle weight in adult rats; this percentage of muscle weight was probably similar in pups, but was not estimated) was removed and the tendons of the muscle were left in situ (Fig. 1C). Care was taken to remove as much of the nerve of the EDL as possible, to avoid the risk of regenerating it into trSol. After surgery, the overlying fascia and the skin were closed. All adult animals were housed with food and water available ad libitum.

EMG electrode implantation. At 3-4 months after muscle transposition, electrodes for chronic EMG recording were implanted in animals of both groups. Under Equithesin anesthesia, six pairs of hook electrodes (AS633; Cooner Wire, Chatsworth, CA) were implanted into muscles: the trSol, gastrocnemius lateralis (GL), and tibialis anterior (TA) in the operated hindlimb, and the TA, EDL, and Sol in the contralateral hindlimb. Other details are as described by Hnik et al. (1978).

EMG recording. At $7 \mathrm{~d}$ after implantation of the electrode array, EMG activity was recorded in awake animals during locomotion and stretch reflexes. Locomotor EMG patterns were recorded during spontaneous locomotion along a straight runway $(2 \mathrm{~m}$ long and $10 \mathrm{~cm}$ wide) (for details, see Slawinska et al., 1995). For each animal the EMG activity was 

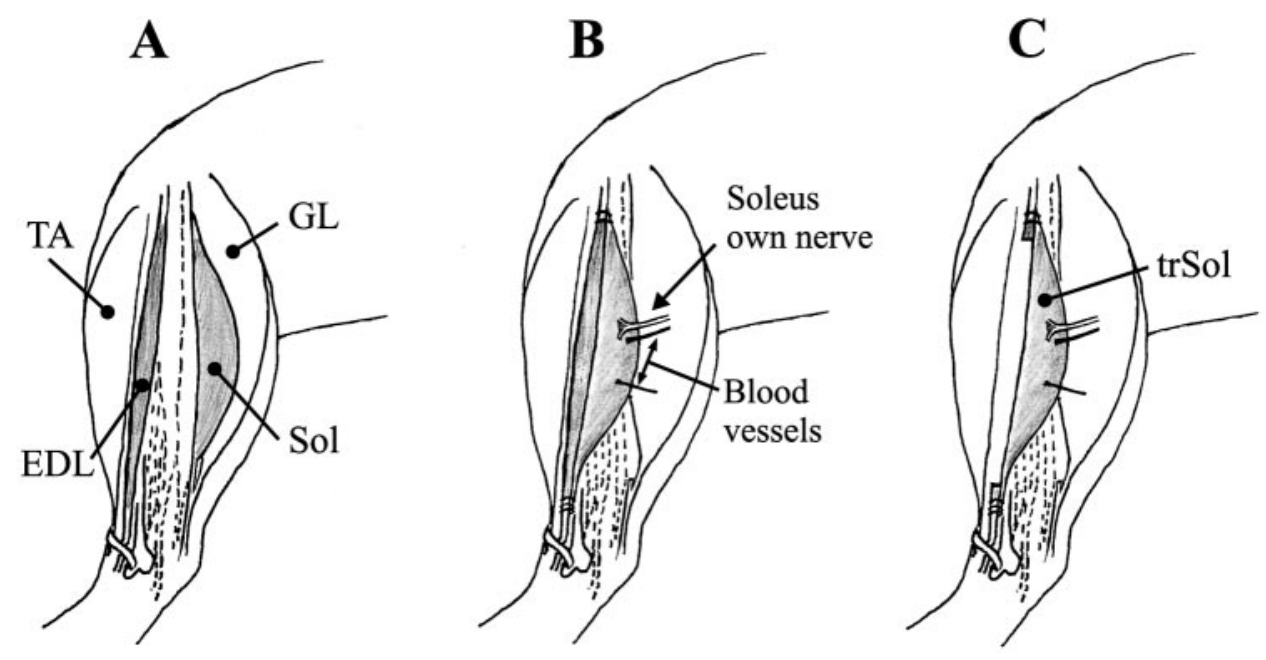

Figure 1. Schematic of consecutive steps of the operative procedure, during which the Sol muscle was transposed into the bed of the EDL muscle. For details, see Materials and Methods. recorded during at least 20 runs, consisting of 8-12 step cycles. Reflex EMG activity was tested by applying passive dorsiflexion and plantar flexion in the ankle. The amplified and filtered EMG activity (bandpass, $0.1-1 \mathrm{kHz}$ ) was recorded on a tape recorder (Racal V-Store; Racal Recorders Ltd., Southampton, UK), digitized off-line (sampling frequency, $2 \mathrm{kHz}$ ), and analyzed using PC software.

\section{Acute experiments}

After completing EMG recordings (4-5 months after muscle transposition), the trSol muscle innervation was verified in acute electrophysiological experiments in six animals from group A. During the experiments, the tension of muscle contraction induced by stimulation of the common peroneal and tibial nerve was recorded. Under chloral hydrate anesthesia $(4.5 \% ; 1 \mathrm{ml} / 100 \mathrm{gm}$ body weight), the common peroneal and tibial nerves in the popliteal fossa were exposed at the distance of $\sim 8-10$ $\mathrm{mm}$ and cut proximally to the spinal cord in the operated and contralateral hindlimbs. The nerve of Sol was also gently exposed. The distal tendons of the Sol (transposed and contralateral) and EDL (contralateral) muscles were dissected free and attached to strain gauges (Harvard Apparatus, Edenbridge, UK). The distal tendons of other surrounding muscles were dissected and cut to avoid the cocontraction influences on tension recordings of investigated muscles. The TA muscle was totally removed. Care was taken not to damage the blood or nerve supply. Isometric contractions were elicited by electrical stimulation of the cut end of the motor nerve supplying the investigated muscles with supramaximal square wave pulses $(50 \mu \mathrm{sec})$ delivered via bipolar silver electrodes. The nerve of the Sol was stimulated to compare its response with the response evoked by stimulation of the nerves in the popliteal fossa. The tension was recorded during single twitch and tetanic contractions $(100 \mathrm{~Hz} ; 600 \mathrm{msec})$ using computer software (bandpass, 0.1-200 Hz; sampling, frequency $99 \mathrm{~Hz}$; Axoscope; Axon Instruments, Foster City, $\mathrm{CA}$ ) and was displayed on the monitor and stored on a hard disk. Before recording, the muscle length was adjusted to obtain maximum twitch tension at the supramaximal stimulus intensity. After tension recordings, the fatigability of the muscle was investigated. A fatigue index was calculated considering the residual tension after 4 min of stimulation at $40 \mathrm{~Hz}$ for $250 \mathrm{msec}$ every second as a percentage of the initial tension. After the experiment, all animals were killed with an overdose of chloral hydrate. The innervation of muscles was inspected under a dissecting microscope, and then the muscles were removed and weighed.

\section{RESULTS}

\section{Chronic experiments}

The general behavior of animals and reflexes were evaluated by visual inspection through the first 3 months after muscle transposition. Because of the immaturity of rat pups in group A, it was difficult to estimate the level of disturbance of locomotor behavior through the first week after surgery. The movements of rat pups were mainly limited to crawling, with the hindquarter of the body dragged on the ground. Visual inspection of spontaneous behavior showed that when standing, the toes of the operated hindlimb were in plantar flexion. When the animal was lifted off the ground by the neck, the operated hindlimb showed only limited flexion at the ankle joint, whereas the toes tended to be flaccid. As distinguished from the control limb, there was no dorsiflexion in the toes of the operated limb. At 4 or $5 \mathrm{~d}$ after surgery, all of these symptoms gradually disappeared. After 1 month, visual inspection of locomotor behavior did not show any clear disturbances in the movements of operated and contralateral hindlimbs.

In all rats operated on as adults (group B), in the first days after surgery the toes of the operated hindlimb were mostly flexed and the heel rested on the ground during standing. When the animals were lifted off the ground, the flexion at the ankle joint and extension of toes was absent in the operated hindlimb. The locomotor limb movements were impaired for $\sim 1$ week after surgery. After 1 month, the locomotor movements improved so that it was impossible to distinguish between the operated and nonoperated hindlimbs by visual inspection. Even during scratching, the animals used either hindlimb without a marked difference in comparison with intact rats. However, in animals that were operated on as adults, the operated leg rested during standing on the ground with the metatarsus only, whereas the toes were in dorsiflexion.

At 3-4 months after muscle transposition, the electromyographic activity of the trSol and other muscles was recorded. Analysis of EMG activity showed that the general postural pattern of activity was preserved. The trSol and control Sol muscles in all experimental animals were continuously active during standing. Activities of the GL, TA (in both hindlimbs), and EDL (in the contralateral hindlimb) were also unchanged (i.e., there was no activity in flexor muscles during standing, but the extensor muscles exhibited tonic activity). When the rat was lifted off the ground, the activity in both Sol muscles disappeared, as in intact animals.

In the intact rat, the activity of flexor (TA, EDL) and extensor (GL, Sol) muscles alternates during locomotion. The trSol muscle in all animals of group A exhibited an additional burst. It occurred during TA activity (i.e., when the Sol muscle in the intact animal was silent) (Fig. 2B, arrows). The results in group B were not uniform. In four animals the trSol presented a normal pattern of activity, typical for extensors; additional bursts occurred in only two animals. In each animal (both from groups A and B) in 




Figure 2. EMG activity of selected muscles during spontaneous locomotion of two rats 3 months after Sol transposition performed in adult $(A)$ and pup $(B)$ animals. In the right column, rectified and integrated EMG activity (normalized and superimposed step cycles; $n=20$ ) of the same muscles is shown. The arrows show additional flexor-like bursts. cSol, Contralateral Sol muscle; $i G L$, GL muscle ipsilateral to trSol; $c T A$, TA muscle contralateral to trSol; $i T A$, TA muscle ipsilateral to trSol; $c E D L$, EDL muscle contralateral to trSol.

which the additional bursts were present, they occurred systematically in all step cycles during slow or fast locomotion.

In all animals, reflex responses were induced by a passive plantar flexion and dorsiflexion at the ankle joint. Under normal conditions, the Sol muscle responds to dorsiflexion, whereas the EDL and TA respond to plantar flexion (Fig. $3 A$ ). During plantar flexion, the trSol was stretched, unlike under normal conditions, when the dorsiflexion causes its lengthening. In five animals from group A, the reflex EMG activity of the trSol was present during both dorsiflexion and plantar flexion of the foot. Plantar flexion evoked not only a typical response of the ankle flexor, TA, but also activated trSol. Dorsiflexion evoked the long-lasting extensor-like activity of trSol, as in the intact Sol (Fig. 3B). In two other animals from group $\mathrm{A}$, the trSol responded to plantar flexion only, which was a typical reflex response of the EDL muscle (Fig. 3C). In all animals from group B, the trSol responded to dorsiflexion, whereas plantar flexion evoked the reflex response in only two animals (in which the additional bursts of trSol occurred during locomotion).

\section{Acute experiments}

The acute experiments were performed on six rats from group A to examine whether the cut end of the EDL nerve (a branch of the common peroneal) also innervated the trSol. In all experiments, electrical stimulation of the common peroneal nerve did not induce any contraction of the trSol. Thus, we concluded that the common peroneal nerve did not innervate this muscle. The response of the trSol to electrical stimulation of the tibial nerve in the popliteal fossa and its branch supplying this muscle proved that Sol innervation survived the muscle transposition.
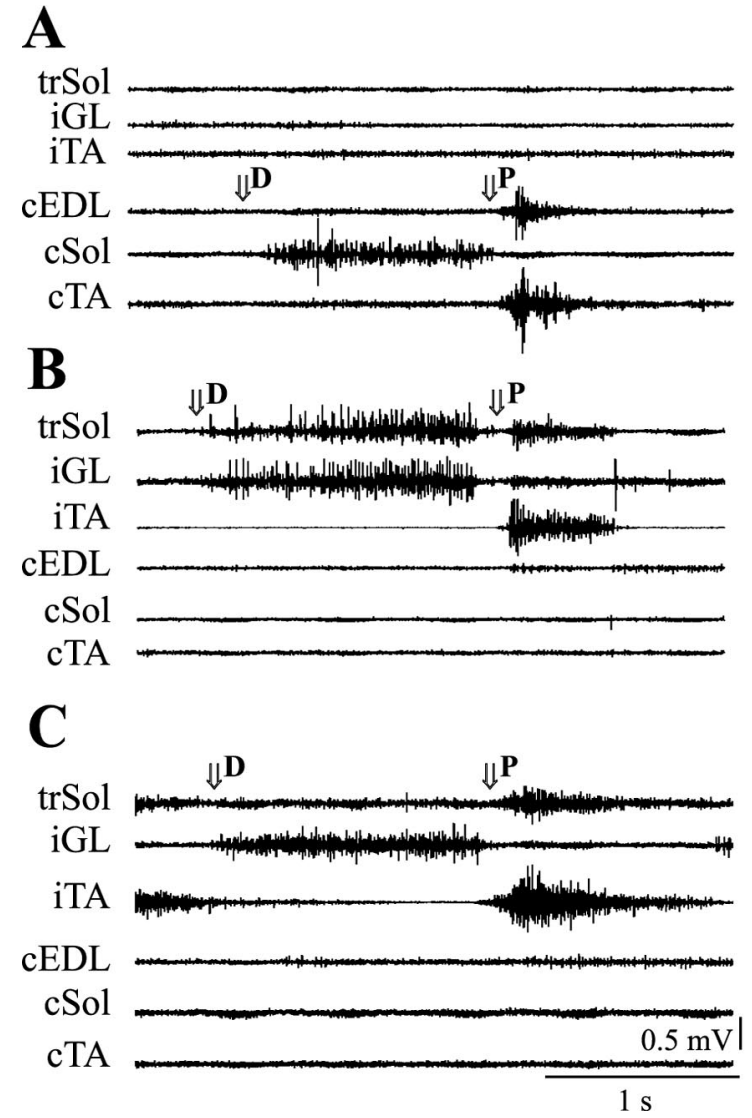

Figure 3. Reflex EMG activity of selected muscles during dorsiflexion $(D)$ and plantar flexion $(P)$ at the ankle joint in two rats from group A. $A$ shows a typical response during dorsiflexion and plantar flexion in a control hindlimb. $B$ shows a partially modified response in an operated hindlimb from the same animal. Note that the trSol responded both to dorsiflexion and plantar flexion. $C$ shows fully modified responses of the trSol in another animal. Note the lack of response in the trSol during dorsiflexion and the clear effect of plantar flexion. The arrows show the beginning of dorsiflexion and plantar flexion. Other denotations are as in Figure 2.

Analysis of muscle tension recordings showed that the trSol was unable to produce a force comparable with that obtained in contralateral intact Sol muscle. In the trSol, the force was three times lower during single twitch contraction (mean \pm SEM; $12.9 \pm 3.1$ vs $33.7 \pm 2.2$ gm; Student's $t$ test; $p<0.001)$ and two times lower during tetanic contraction (mean \pm SEM; $108.3 \pm$ 30.6 vs $207.2 \pm 22.7 \mathrm{gm}$; Student's $t$ test; $p<0.03$ ) compared with contralateral intact Sol muscle. This means that the trSol was much weaker than the intact Sol. The other indices (the time to peak in a single twitch and fatigability) for trSol did not differ from the control muscle (mean \pm SEM; $69.7 \pm 4.6$ vs $63.0 \pm 4.5$ msec and $90.4 \pm 4.8$ vs $79.6 \pm 8.4 \%$, respectively; Student's $t$ test; $p>0.05$ for both). This means that $4-5$ months after surgery, the properties of the trSol did not change and the muscle remained as slow and fatigue resistant as intact Sol. The weight of the trSol remained at the same level as that of control muscle (mean \pm SEM; $190 \pm 30$ vs $220 \pm 30 \mathrm{mg}$; Student's $t$ test; $p>0.5)$.

Postmortem visual inspection under the dissecting microscope showed that all trSol muscles survived the transposition in good condition. The shape of the Sol muscle transposed in immature rats resembled the fusiform EDL muscle (i.e., it was thinner in the middle part than normal Sol). The innervation of the trSol orig- 
inated in the tibial nerve, and any other innervation of the transposed muscle was not detected.

\section{DISCUSSION}

Our results showed that the locomotor and reflex activity EMG pattern of the Sol might be modified when the muscle is transposed into the bed of an antagonistic muscle, the EDL. To exclude the possibility that the flexor-like activity was induced by additional innervation of the transposed muscle by the cut end of the EDL nerve, we also performed acute experiments. Electrical stimulation of the common peroneal nerve did not induce any contraction of the trSol. Thus, we conclude that the observed modification of muscle activity was indeed the result of changes in the organization of spinal network connections.

The functional rearrangement of the transposed muscle activity was better manifested when the transposition was done in immature rats (6-d-old pups), although some changes also occurred in one-third of animals operated on as adults. This phenomenon may be based on several factors. The most substantial might be the immaturity of the neuromuscular system at the moment of transposition and preservation of sensory/motor innervation of the transposed muscle. The second factor might be substantial for changes observed in animals operated on as 3-month-old adults.

The neuromuscular system of the rat at the moment of birth is at a relatively immature stage. The Sol muscle is activated phasically until day 12, and then develops the adult tonic activation pattern (Navarrete and Vrbova, 1983). The polyneuronal innervation pattern of the Sol is reduced to the adult pattern during the second half of the second postnatal week (Brown et al., 1976), and the firing pattern of spindles reaches adult characteristics between days 14 and 18 (Vejsada et al., 1985). Maturation of the neuromuscular system enables the development of posture and movement abilities. Behaviorally, from day 4 or 5 until day 12 or 13, the pups walk with staggering motion. The adult type of locomotion is present after day 16 (Geisler et al., 1993).

Successful transformation of the activity of the trSol occurred in all animals from group A, which were operated on when the neuromuscular system was immature. It is likely that preservation of the sensory/motor innervation of transposed muscle provided a physical substrate, enabling neural networks to continue the maturation process and adaptation to a modified situation, depending on afferent signals from muscle spindles and Golgi receptors. During locomotion, the central pattern generator (CPG) network activated the trSol simultaneously with its agonistic extensor muscles (i.e., trSol actively contracted, but at the same time it was passively lengthened). Because some of the Golgi receptors might survive the transposition, activation of these receptors may partly inhibit the activity of trSol motoneurons during the stance phase. In contrast, at the beginning of the swing phase the afferent signals from muscle spindles in the trSol might facilitate its activation, because the muscle was stretched to maximal length at the end of the stance phase. A combination of these signals may cause functional "tuning" of the transposed muscle to the new situation.

The reorganization of spinal circuitry was also proved by the results of an investigation of spinal reflexes. Dorsiflexion at the ankle joint under normal conditions induces the activation of the Sol and other agonistic extensor muscles. Such an activation of trSol, although it was not stretched, was present in all animals from group $\mathrm{B}$ and most animals from group A. Thus, we think that this activation was a "shadow" of extensor reflex activity evoked by afferent signals from the intact, homonymous ankle extensors. The plantar flexion in intact animals stops the activity of extensors induced by dorsiflexion and induces the activation of flexor muscles. The trSol was stretched during plantar flexion, as were other flexors, so one could expect that it should respond to stretch. Such a reaction was present in all of the animals from group A but only in two animals from group B. Stretching flexor muscles during plantar flexion induces the excitation of these muscles and simultaneous inhibition of extensor muscles. At the same time, stretching of the trSol induces excitation of its motoneurons. The final response of trSol is the result of summation of both inhibitory and excitatory inputs. Different responses in both groups indicate that alteration of connections within the reflex pathways was more pronounced in animals operated on at a young age rather than as adults. The possibility of spinal network reorganization after neonatally transferred tendon in rats was also shown for the nociceptive reflex pathways (Holmberg et al., 1997).

One problem, to what extent the functional readjustment may take place in the spinal cord, is still open. Humans can learn how to adapt to muscle transposition in the upper or lower limb. In lower mammals, it still remains the problem of plasticity in neural nets after tendon transfer. The CPG in the cat seems to be more "hardwired" (Forssberg and Svatengren, 1983), although there are data demonstrating that it is capable of learning and adaptation (Loeb, 1999). We found in the rat that the pattern of transposed muscle activity was rearranged in line with new functional demands after transposition, although the innate Sol activity was still preserved. However, analysis of the EMG activity cannot help to determine at which level the plasticity takes place. It is possible that transposition of the Sol changed the balance of afferent inputs to motoneurons and the CPG network that is responsible for the generation of the locomotor pattern.

The results we obtained for group B are not uniform. Although the additional burst of trSol occurred during locomotion in two cases, in four other cases it was absent. The latter result is consistent with the work of Sperry (1940), who did not find any sign of adaptation after transposition of hindlimb muscles in the adult rat. A partial explanation of the difference could be the fact that transferring antagonistic muscles to opposite positions at the same time did not modify the mutual relationships among them, because it did not change the relationships among transferred muscles after surgery; the muscles remained antagonistic. Moreover, by removing many other muscles, Sperry (1940) eliminated most of the afferent signals from the operated limb. Preserved muscles were activated in a typical sequence by CPG activity and received characteristic afferent signals from themselves and from antagonistic muscle. Transposed muscles did not "know" that their activity was out of phase in relation to the swing/stance locomotor pattern because there were no afferent signals from other muscles.

In our case, with other preserved muscles, activation of trSol during the stance phase caused some perturbations to the limb movements. The muscle tends to evoke flexion at the ankle when extension is expected. As a result, the pattern of afferent signals from the transposed muscle, as well as from other muscles, differs from the normal ones. These changes may be followed by the readjustment of connections within the spinal cord circuitry. According to the pattern of muscle activity, the rearrangement could consist of making new connections or of changing the weight of the synapses responsible for the generation of additional bursts, which was easier in immature animals.

Functional reorganization of hindlimb movements after cross- 
reinnervation has been described in the monkey (Sperry, 1947). Results on rats and cats operated on in a similar manner were negative or unclear (Sperry, 1940; Luff and Webb, 1985; Gordon et al., 1986). In terms of movement patterns, the functional modification in lower mammals was reflected by incomplete movement reversal. Muscles were initially activated according to the normal pattern of activity of the antagonist nerve that supplied them, and the animals made corrective maneuvers after that to ensure continued progression. The functional modification observed after cross-reinnervation of hindlimb extensor and flexor muscles in cats was probably attributable to the discrepancy between the efferent signal from the CNS and the functional demands of cross-reinnervated muscles.

It is well known that there are two basic types of movements: reflex (innate) and voluntary. The voluntary movements are controlled by central mechanisms and must be learned, although when established they can be generated without peripheral feedback (Polit and Bizzi, 1979). The process of learning, during which such movements are elaborated, implies a central plasticity. The muscle with preserved innervation transposed at an early stage of development should be more easily incorporated into a new pattern of activity. The locomotor movements controlled by the CPG network (Grillner and Zangerr, 1979) can be driven by signals from higher centers and modified by a peripheral feedback to adapt the activity pattern to the environmental conditions. Our results show that transposition of extensor muscle into the bed of flexor, performed in immature animals, may alter the locomotor activity pattern generated by spinal networks. To some extent this phenomenon was also observed after muscle transposition performed in adult rats. It is difficult to say whether this alteration was attributable to changes in supraspinal signals, reorganization of spinal circuitry, or modifications of spinal reflex pathways. It is interesting that the additional flexor-like burst that is present during locomotion occurred in animals in which plantar flexion evoked a reflex response in the trSol. In our opinion, it is likely that these changes are attributable to modifications at the spinal level, although on the basis of the results presented it is difficult to prove this hypothesis. Additional experiments on the spinal or deafferented animals are needed to solve the problem.

In summary, we found that the activity of trSol muscle in immature animals may be classified during various movements as flexor- or extensor-like, depending on the actual direction of the movement in the ankle joint. We interpret the readjustment to the new functional demands (e.g., an extra burst of trSol muscle during the ankle flexion) as plastic change. The plasticity of the neuromuscular system was more effective in animals that were immature at the moment of muscle transposition. It remains to be determined whether the mechanism of the observed plasticity was based on changes of the afferent input from the Sol (periphery dependent) or whether these changes evoked some "rewiring" of the CPG network.

\section{REFERENCES}

Brown MC, Jansen JK, Van Essen D (1976) Polyneuronal innervation of skeletal muscle in new-born rats and its elimination during maturation. J Physiol (Lond) 261:387-422.

Forssberg H, Svatengren G (1983) Hardwired locomotor network in cat revealed by a retained motor pattern to gastrocnemius after muscle transposition. Neurosci Lett 41:283-288.

Geisler HC, Westerga J, Gramsbergen A (1993) Development of posture in the rat. Acta Neurobiol Exp (Warsz) 53:517-523.

Gordon T, Stein RB, Thomas CHK (1986) Innervation and function of hind-limb muscles in the cat after cross-union of the tibial and peroneal nerves. J Physiol (Lond) 374:429-441.

Grillner S, Zangerr P (1979) On the central generation of locomotion in the spinal cat. Exp Brain Res 34:241-262.

Hnik P, Kasicki S, Afelt Z, Vejsada R, Krekule I (1978) Chronic polyelectromyography in awake, unrestrained animals. Physiol Bohemoslov 27:485-492.

Holmberg H, Schouenborg J, Yu Y-B, Weng H-R (1997) Developmental adaptation of rat nociceptive withdrawal reflexes after neonatal tendon transfer. J Neurosci 17:2071-2078.

Loeb GE (1999) Asymmetry of hindlimb muscle activity and cutaneous reflexes after tendon transfers in kittens. J Neurophysiol 82:3392-3405.

Luff AR, Webb SN (1985) Electromyographic activity in the crossreinnervated soleus muscle of unrestrained cats. J Physiol (Lond) 365:13-28.

Missiuro W, Kozlowski S (1963) Investigation on adaptive changes in reciprocal innervation of muscles. Arch Phys Med Rehabil 44:37-41.

Navarrete R, Vrbova G (1983) Changes of activity patterns of slow and fast muscles during postnatal development. Brain Res Dev Brain Res 8:11-19.

Polit A, Bizzi E (1979) Characteristics of motor programs underlying arm movements in monkey. J Neurophysiol 42:183-194.

Slawinska U, Navarrete R, Kasicki S, Vrbova G (1995) Motor activity patterns in rat soleus muscle after partial denervation. Neuromuscul Disord 5:179-186.

Sperry RW (1940) The functional results of muscle transposition in the hindlimb of the rat. J Comp Neurol 73:379-404.

Sperry RW (1947) Effects of crossing nerves to antagonistic limb muscles in the monkey. Arch Neurol Psychiat (Chic) 58:452-473.

Vejsada R, Hnik P, Payne R, Ujec E, Palecek J (1985) The postnatal functional development of muscle stretch receptors in the rat. Somatosens Res 2:205-222.

Yumiya H, Larsen KD, Asanuma H (1979) Motor readjustment and input-output relationship of motor cortex following crossconnection of forearm muscles in cats. Brain Res 177:566-570. 